\title{
AFM-Imaging Diagnosis Method for Single Nucleotide Polymorphism Using Molecular Beacon DNA as an Intramolecular Ligation Template of Target DNA and a Viewable Indicator
}

\author{
Hisao Yoshinaga, ${ }^{*}$ Koji NaKano, ${ }^{* \dagger}$ Nobuaki SoH, ${ }^{* *}$ and Toshihiko Imato* \\ *Department of Applied Chemistry, Faculty of Engineering, Kyushu University, 744 Motooka, Nishi, \\ Fukuoka 819-0395, Japan \\ **Department of Agriculture, Saga University, 1 Honjo, Saga 840-8502, Japan
}

\begin{abstract}
An AFM-imaging-based method for single nucleotide polymorphism (SNP) analysis is described. A stem-loop-forming 34-mer oligonucleotide (p34s) was designed. P34s contains the complementary sequence for K-ras (5'-GGT GGC-3', t6G), one of the human oncogenes, at the $5^{\prime}$-end for target-recognition and five successive phosphorothioate linkages in the loop. The functional probe, either alone or hybridized with target DNA (p34s/t6G), relaxed upon treatment with "opener" DNA. The template/target DNA interstrand hybridization product is covalently connected by ligase if the correct target is used, but not hybridized species including mismatches. With these results, developed was a solid-phase SNP assay by transferring an aliquot of the product onto an $\mathrm{Au}(111)$ substrate for self-assembly, followed by AFM imaging. Clear contrasts that allow the detection of SNPs, were observed for the ligated and non-ligated species representing the loop-to-linear conformational change. Simple statistical surface-roughness analysis determined the lowest concentration of the sample to be $5 \times 10^{-10} \mathrm{M}$, whose necessary sample quantity was 5 fmol.
\end{abstract}

(Received July 18, 2012; Accepted August 22, 2012; Published October 10, 2012)

\section{Introduction}

Intelligent readouts of chemical binding equilibria or reaction kinetics are major objectives for most biomacromolecules when developing corresponding analysis methods. To date, small molecule reporters that send responses to a transducer device provide a convenient means of probing the dynamics of biomacromolecules; the response of molecular oxygen to glucose oxidase is a typical example. Concerning nucleic acids, fluorophore labels have succeeded the long-standing radioisotope labels, and have been producing good results, because they are versatile enough to be used in a large body of applications ranging from simple biosensors to modern high-throughput analytical devices. ${ }^{1}$ Recent developments in nanotechnology, on the other hand, provide opportunities for advanced biosensing. In particular, scanning probe microscopy (SPM), which is a versatile surface observation method, is anticipated to allow biomolecules to be imaged and examined down to the single-molecule level. ${ }^{2}$ Most biomacromolecules inevitably undergo higher-order structural changes when they exert their biocatalysis functions. Thus, by including a molecular mechanism that characterizes these subtle differences, SPM will provide molecular read-out capability to build a strong framework for advanced biosensing.

At present, the accomplishment of The Human Genome Project has stimulated broad interest in identifying specific genes that cause genetic diseases. Certain types of solution-

$\dagger$ To whom correspondence should be addressed.

E-mail: nakano@cstf.kyushu-u.ac.jp phase assays are being examined for migration into solid-phase testing devices to establish simple, fast, portable, and sensitive diagnosis methods. Molecular beacon (MB) $\mathrm{DNA}^{3}$ belongs to this category, and are employed in genetic screening, ${ }^{4}$ biosensors, ${ }^{5,6}$ single nucleotide polymorphism (SNP) analysis, ${ }^{7,8}$ and mRNA monitoring. ${ }^{9}$ Other applications in solid-phase assays taking advantage of fluorophores or electrochemical labels have also been established. ${ }^{10,11}$ Until now, small molecule functional labels have been principally included in MB design to produce readable signals upon transformation from stem-loop to double-helical structures that is caused upon DNA molecular recognition. In the present study, we achieved direct visualization of a MB DNA probe, including its stem-loop-to-linear conformational changes. Moreover, the AFM imaging-based method was successful in detecting SNP for K-ras, one of the human oncogenes.

\section{Experimental}

Biochemical materials and chemical reagents

ODN sequences were obtained from a custom synthesis by Sigma Genosys (Sapporo, Japan), and were used as received (Table 1). They include stem-loop-forming sequences, p34s (probe DNA) and c40s (positive control); target DNA, t6G; single-mismatch DNA sequences (negative controls), t6A, t6C, and t6T; loop-complementary DNA (opener), o8. The underlined bases are self-complementary to form an intracatenary double helical structure. The mfold- ${ }^{12}$ and OligoCalc-softwares ${ }^{13}$ predicted the stem-loop structure and $T_{\mathrm{m}}$, respectively. The s-notation (As, Cs, and Gs) represents a phosphorothioester linkage. 
Table 1 Base sequences of DNA

\begin{tabular}{ll}
\hline Abbr. & \multicolumn{1}{c}{ Sequence $\left(5^{\prime}\right.$ to $\left.3^{\prime}\right)$} \\
\hline p34s & $\underline{\text { GCCACC-GCGAGG-TAAAsAsCsGsAsC-GGCCAGT- }}$ \\
c45s & $\underline{\text { GCTCGC }}$ \\
& CCTCC-GCGAGG-TAAAsAsCsGsAsC-GGCCAGT- \\
t6G & $\underline{\text { GGTGGC }}$ \\
t6A & $\underline{\text { AGTGGC }}$ \\
t6C & CETGGC \\
t6T & TETGGC \\
o8 & GACTGGCC \\
\hline
\end{tabular}

The tetramethylrhodamine (TAMRA)-labeled ODN, p34sF, was used for fluorescence energy-transfer measurements, combined with the use of a quencher (black hole quencher-2, BHQ2)labeled ODN, t6GQ or t6AQ. Each ODN was dissolved in tris-ethylenediaminetetraacetic acid buffer (TE buffer, $10 \mathrm{mM}$ Tris- $\mathrm{HCl} / 1 \mathrm{mM}$ ethylenediaminetetraacetic acid, $\mathrm{pH}$ 8.0) to give a solution with a concentration of $100 \mu \mathrm{M}\left(1 \mathrm{M}=1 \mathrm{~mol} \mathrm{dm}^{-3}\right)$. In the cases of p34s and $\mathbf{c 4 0 s}$, the ODN solution was annealed $\left(0.5^{\circ} \mathrm{C} \mathrm{min}-1\right)$ prior to use. The ligation reaction was performed using T4 DNA ligase with a DNA ligation kit (Mighty Mix ${ }^{\circledR}$, Takara Bio Inc., Japan). All chemical reagents were guaranteed reagents and were used as received.

\section{Template reaction using T4 DNA ligase}

Solutions of p34s and t6G $(20 \mu \mathrm{M}, \mathrm{pH} 8.0$ Tris- $\mathrm{HCl}$ containing $300 \mathrm{mM} \mathrm{NaCl}$ and $5 \mathrm{mM} \mathrm{MgCl}_{2}, 100 \mu \mathrm{L}$ ) were mixed, annealed at $80^{\circ} \mathrm{C}$ for $10 \mathrm{~min}$, and then cooled at a rate of $0.5^{\circ} \mathrm{C} \mathrm{min}-1$ down to $10^{\circ} \mathrm{C}$. A solution of T4 DNA ligase $(50 \mu \mathrm{L})$ containing enzyme ( $2 \mathrm{nmol})$ was added, and then the solution was maintained for $12 \mathrm{~h}$ at $15^{\circ} \mathrm{C}$ to allow equilibration. The ligation reaction was performed using a DNA ligation kit, Mighty Mix ${ }^{\circledR}$ (Takara Bio Inc.). This was typically followed by ethanol precipitation to allow DNA purification.

\section{Characterization of DNA self-assembly}

Polished glass (Tempax, $18 \times 18 \mathrm{~mm}$ ) with an evaporated $\mathrm{Au}$ film was used as a substrate. After pretreatment, the substrate was treated with the probe DNA solution p34s $(5 \mu \mathrm{M}, 150 \mu \mathrm{L})$ for $24 \mathrm{~h}$ at room temperature by a simple solution casting method. Fourier-transform infrared reflection absorption spectroscopy (FTIR-RAS) was performed on a FTIR620 spectrometer (JASCO Co., Japan). The $p$-polarized irradiation was introduced to the sample at an angle of $85^{\circ}$ off the surface normal, and data were obtained at a spectral resolution of $1 \mathrm{~cm}^{-1}$ with 256 scans. The thickness of the p34s self-assemblies was analyzed on an imaging ellipsometer (Japan Laser Co., Japan) equipped with a He-Ne laser $(5 \mathrm{~mW}, 632.8 \mathrm{~nm})$ as a light source. Reported values are the average of measurements obtained in 16 zones. Microgravimetric analysis was performed using a QCA917 quartz crystal microbalance (QCM, Seiko EG\&G Co. Ltd., Japan). Changes in frequency upon ODN chemisorption were collected on AT quartz crystals (fundamental frequency $9 \mathrm{MHz}$ ) possessing a pair of evaporated $\mathrm{Au}$ electrodes (geometrical area, $0.196 \mathrm{~cm}^{2}$; roughness factor, 1.6). As reported by Schlenoff and his coworkers, the surface morphology of the substrate was not the primary concern for alkanethiol selfassembly ${ }^{14}$ and we followed the empirical results. More detailed experimental procedures can be seen in a previous report. ${ }^{15}$

\section{AFM imaging and SNP assay}

The p34s hybridization products obtained from four different kinds of target DNA were subjected to the T4 DNA ligase reaction, described above. In the case of ring-opening, an equimolar amount of $\mathbf{0 8}$ dissolved in TE buffer was added to the reaction mixture. For a comparison, all ODN conjugate species shown in Fig. 1 were prepared separately and examined by AFM. Each DNA conjugate solution $(10 \mu \mathrm{L})$ was cast on an evaporated gold film with a (111) texture (Agilent Technologies, Inc., CA). The sample solution typically formed a water droplet with a diameter of $c a .3 \mathrm{~mm}$ on the gold surface. The substrate was then stored in an airtight vessel to prevent drying, and left to self-assemble for $24 \mathrm{~h}$. Molecular imaging of the DNA samples was performed using a JSPM-4210 or JSPM-5410 scanning probe microscope (JEOL, Tokyo, Japan). The instrument was operated in non-contact mode imaging with frequency modulation detection. All AFM images were obtained by $512 \times 512$ pixels, and the nearest point that the cantilever tip could approach, while scanning, was chosen as the reference for the corresponding surface height image. Simple low-path filtering using a Fourier transform reduced the noise, which was provided by either the Winspm II data-processing software (JEOL, Tokyo, Japan) or the Image SXM. ${ }^{16}$ Silicon cantilevers $\left(k=14.0 \mathrm{~N} \mathrm{~m}^{-1}, f_{0}=315 \mathrm{kHz}, \quad r=10 \mathrm{~nm}\right)$ purchased from Mikromasch (Tallinn, Estonia) were used throughout the study.

\section{Results and Discussion}

\section{Molecular design of MB DNA as a SNP probe}

We have used AFM to image MB DNA self-assemblies; its molecular resolution revealed for the first time that double helices could be discriminated from extended loops in surface images obtained at high resolution. ${ }^{17}$ We expand the use of MB DNA to SNPs detection by taking advantage of rational probe DNA design; the indicator DNA probe potentially forms a stem-loop structure through intramolecular base pairing, and simultaneously consist of a template sequence and a reactive site which renders surface-active. The DNA sequences (Table 1) include indicator DNAs, p34s and c40s (positive control), both of which contain five-successive phosphorothioate linkages (s-oligo); target DNA, t6G that represents the codon $12-13$ in K-ras; single-mismatch DNA sequences (negative controls), t6A, t6C, and t6T, some of which are the cause of various malignancies and carcinomas; loop-complementary DNA (opener), o8. Figure 1 summarizes the methodology of label-free detection. The mfold, ${ }^{12}$ a web-based nucleic acid folding software program, predicted that the native form of $\mathbf{p 3 4 s}$, i.e., the phosphodiester form of $\mathbf{p 3 4 s}$, will fold into a stem-loop conformer, forming hydrogen bonds at the intracatenarycomplementary pairs (the underlined sequence). The empirical prediction program OligoCalc ${ }^{13}$ predicted that the resulting stem-loop, when produced from the complementary hexamerpair, would exhibit a $T_{\mathrm{m}}$ of $22^{\circ} \mathrm{C}$. A simple comparison of the $T_{\mathrm{m}}$ values also suggested that the binding of $\mathbf{0 8}$ at the corresponding sequence in the loop (the wiggly-lined sequence) will relax the structure (Fig. 1 path 1 and $2 b$ ), because the resulting p34s/o8 duplex is more stable $\left(T_{\mathrm{m}}=28^{\circ} \mathrm{C}\right)$ than the hexamer loop. The extra sequence at the $5^{\prime}$-end (marked with the double-underline) in $\mathbf{p 3 4} \mathbf{s}$ could assist the ligase reaction by acting as a template. After the ligase reaction, the enhanced stability of the resulting p34s-t6G covalent conjugate $\left(T_{\mathrm{m}}=\right.$ $44^{\circ} \mathrm{C}$ ) can prevail against the ring-opening stresses induced by the binding of $\mathbf{0 8}$ (path 2a). The ligation reaction only occurs with fully matching target DNA, whereas leaves the mismatch 


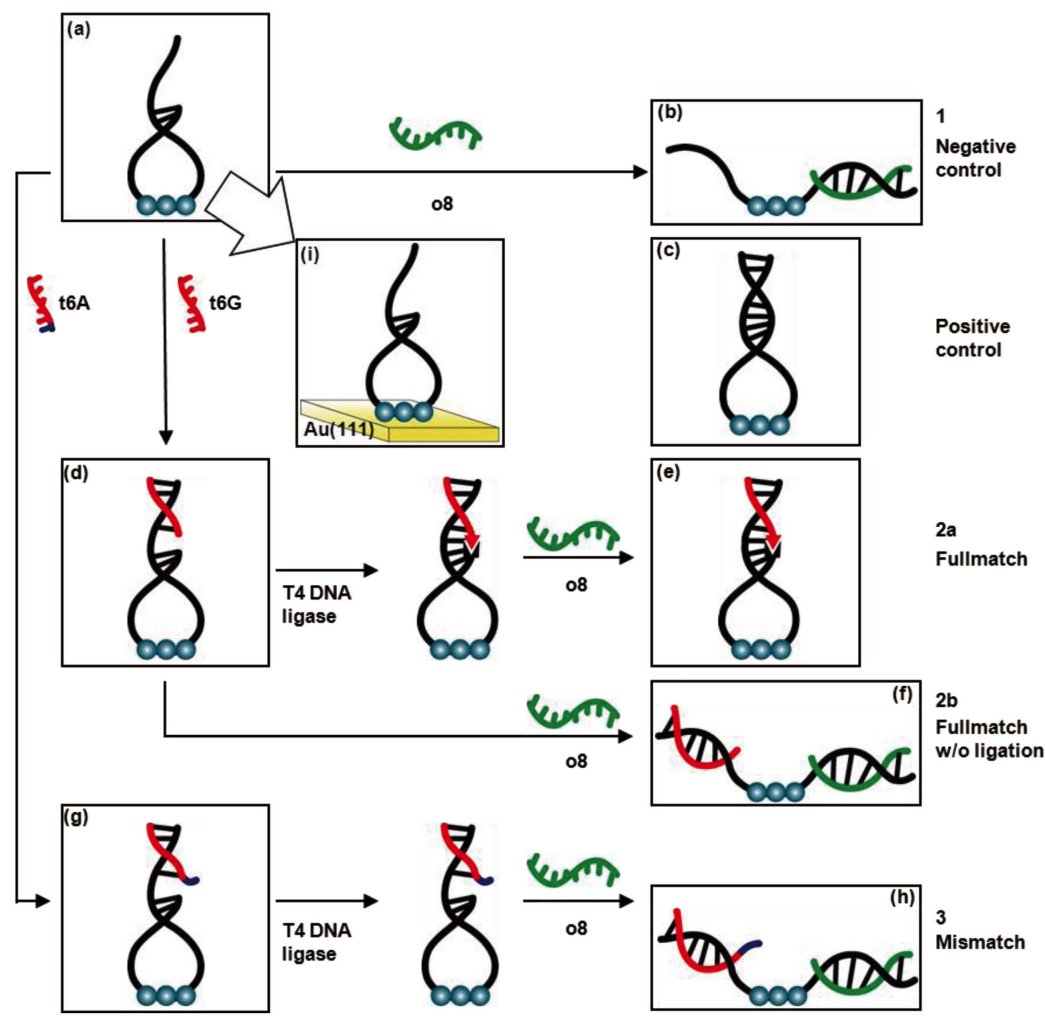

Fig. 1 Illustration of the AFM based experiment of single nucleotide polymorphism assay and conceptualistic images of $\mathrm{Au}(111)$ substrates after developing each MB self-assembly containing $\mathbf{p 3 4 s}$ (a), after hybridization of p34s and $\mathbf{0 8}$ (b), control probe of c40s (c), p34s and t6G (d), after ligation of p34s and t6G, and hybridization with 08 (e), p34s and t6G, and hybridization with 08 (f), p34s and t6A (g), after ligation of p34s and t6A, and hybridization with $\mathbf{~} \mathbf{8}(\mathrm{h})$. Each MB species was self-assembled on the $\mathrm{Au}(111)$ substrate for AFM imaging (i).
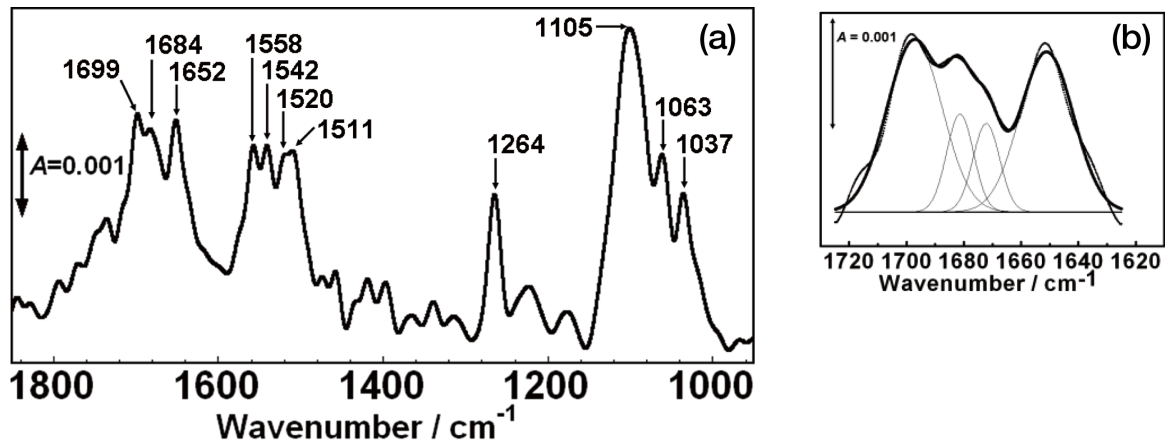

Fig. 2 FTIR reflection-absorption spectrum of p34s immobilized on an Au substrate (a), and the quantitative curve-fitting analysis for the carbonyl groups in $\mathbf{p 3 4 s}$ (b). In (b), results for the quantitative curve-fitting analysis for the $-\mathrm{C}=\mathrm{O}$ region are shown in thin lines, while their sum total (thick line) reproduces the original data (filled circle) well. The assignment of these bands is characterized $1699 \mathrm{~cm}^{-1}$ as a guanine group, 1684 and $1672 \mathrm{~cm}^{-1}$ as a thymine group, and $1652 \mathrm{~cm}^{-1}$ as a cytosine group, respectively.

samples unlinked with $\mathbf{p 3 4 s}$ to enable discrimination of them by $\mathbf{0 8}$ treatment (path 3). Fluorescence energy-transfer measurements inherently confirmed the working principle (Figs. S1 and S2, Supporting Information).

\section{Characterization of p34s self-assembly on Au surfaces}

For our AFM-based method to become workable, we must attach the probe DNA and its target-DNA-hybrid properly on solid supports. Previously, we reported that a s-oligo modification at the backbone phosphodiester linkage is effective for self-assembly on Au surfaces. ${ }^{18,19}$ Therefore, the indicator DNA sequence was partly converted into the phosphorothioate structure.

FTIR-RAS measurements confirmed that the formed surface phases reflected the intended adsorbates. Figure 2 shows a representative FTIR-RAS spectrum for a p34s-treated gold 
substrate. By reference to the structural features of nucleic acids, two peaks at 1264 and $1105 \mathrm{~cm}^{-1}$ were assigned as antisymmetric and symmetric $\mathrm{P}-\mathrm{O}$ stretches of the phosphate group, respectively. The peak at around $1050 \mathrm{~cm}^{-1}$ was identified as the deoxyribose $\mathrm{C}-\mathrm{O}-\mathrm{C}$ stretch of the backbone. A broad band consisting of components at 1558, 1542, 1520, and $1511 \mathrm{~cm}^{-1}$ is assigned as the absorption of the purine skeleton. Lastly, the intense peaks between $1700-1650 \mathrm{~cm}^{-1}$ are ascribed as $\mathrm{C}=\mathrm{O}$ stretches. Theoretical waveform analysis showed that this particular absorption band could be further deconvoluted to give four-distinct absorptions at 1699, 1684, 1672, and $1652 \mathrm{~cm}^{-1}$, which are assigned as the $\mathrm{C}=\mathrm{O}$ stretches of guanine, thymine (two bands), and cytosine, respectively.

Next, ellipsometry was used to determine the thickness of the p34s molecular aggregate. For analysis, an air-organic film-substrate three-layer model was adopted. Using an iterative procedure (least-square minimization), unknown optical constants and/or thickness parameters were varied to determine the parameter set that best matched the experimental data. A reflective index of 1.45 was assigned for the $\mathbf{p 3 4 s}$ self-assembly, which seems to be reasonable for an organic film. The thickness of the film was determined to be $7.2 \pm 0.7 \mathrm{~nm}(n=16)$. CoreyPauling-Koltun (CPK) model building estimated that the thickness of the extended stem-loop structure without the singlestranded recognition sequence was $6.1 \mathrm{~nm}$. For the singlestranded recognition sequence, an extra $1.2 \mathrm{~nm}$ can be added. The estimated molecular height is consistent with the film thickness data determined by ellipsometry. Moreover, the agreement of these values allows us to conclude that p34s adopts a vertically aligned orientation at the substrate surface.

Finally, microgravimetric analysis using a QCM was used to determine the amount of $\mathbf{p 3 4} \mathbf{s}$ immobilized on the substrate surface. A frequency decrease of $\Delta F=-165 \pm 33 \mathrm{~Hz}$, which is equivalent to a surface concentration of $27 \pm 5.8 \mathrm{pmol} \mathrm{cm}^{-2}$ $(n=6)$ of the ODN, was found using the Sauerbrey equation. CPK model building showed the footprint of the five successive nucleotides to be $4.2 \mathrm{~nm}^{2}$, which indicates a theoretical surface concentration of $39 \mathrm{pmol} \mathrm{cm}^{-2}$. By comparing these values, we can conclude that a surface coverage of $70 \pm 15 \%$ is attained upon the chemisorption of p34s. From these results, we can conclude that the phosphorothioate group in the $\mathbf{p 3 4 s}$ probe undergoes near self-assembly on Au substrates to fix the whole molecule entity on the Au surface. Because QCM analysis showed that the native form of $\mathbf{p 3 4} \mathbf{s}$ did not give any resonantfrequency response, the phosphorothioate functions in the ODN are acknowledged to play a pivotal role in self-assembly.

\section{Detection of SNP in the K-ras gene based on AFM imaging}

Combining the above-mentioned results, we examined an AFM-imaging-based SNP detection by transferring an aliquot of the product onto an $\mathrm{Au}(111)$ substrate. In short, molecular visualization turned out that every solution species shown in Fig. 3 gave a specific AFM image; all of the surface images consisted of either compact, hemispheres or extended rope-like motifs. Neither the untreated bare Au substrates nor those treated with the native form of p34s gave such kinds of molecular images. The results are summarized as follows.

The p34s self-assemblies produced one of two types of unambiguous images (Fig. 3a); physical matter is finely distributed almost completely over the substrate surface. They are commonly found to be compact hemispheres with a diameter of $c a .10 \mathrm{~nm}$. Because the loop is chemisorbed to the substrate surface, the structurally flexible recognition sequence is outward facing, and this may blur the AFM image. The large diameter of these entities in anomalous for a single DNA molecule, and may be caused by destruction of the tip apex. Together with the spectroscopy and QCM data, we can conclude that each surface entity must be an individual p34s molecule.

Hybridization with $\mathbf{0 8}$ before self-assembly definitely produced a different kind of surface image (Fig. 3b); one can see a fully stretched, linear motif over the entire substrate surface. It is believed that each motif represents a single p34s molecule that has a specific length of $24 \mathrm{~nm}$ in its ladder conformation. The $\mathbf{0 8}$ binding also characterized the stem-loop to the linear conformation transition of $\mathbf{p 3 4} \mathbf{s}$ after treatment with the complementary, full-match target t6G (Fig. 3d), or with that having single mismatch, t6A (Fig. 3g). Interestingly, T4 DNA ligase successfully contrasted the two different products containing target DNA; T4 DNA ligation maintained the compact hemispheres of the $\mathbf{p 3 4}$ / complementary target conjugate (Fig. 3e), while samples including mismatches changed the images reliably (Fig. 3h). As positive control, c40s self-assemblies gave images of compact hemispheres (Fig. 3c). In addition, when the $\mathbf{p 3 4} \mathbf{s} /$ complementary target conjugates was left the unligated form, observed were the fully stretched, linear motifs (Fig. 3f). The same experiments using single-base mismatched target DNA strands t6C and t6T also gave similar images to (h) (results not shown). Contrary to RNAs that adopt very unique conformations through inter- and intra-strand base pairing, ${ }^{20}$ the stiff-chains of DNA duplexes could exclude various microscopically distinctive structures other than the hemispheres or ropes; they correspond to the molecular views along with either the short axis or the long-axis of the duplex. Indeed, self-assembly of the indicator DNA at the phosphorothioate sequences could contrast the hemisphere against the rope; the double-helical portion adopts a perpendicularly oriented structure, whereas the liberated linear form aligns horizontally at the substrate surface. We can conclude that AFM-imaging of the MB DNA self-assembly offers a potential detection method of SNPs, when combined with the template reaction using T4 DNA ligase.

\section{Surface roughness analysis and sensitivity of detection}

Finally, the sensitivity of the detection of the surface morphology-based SNP assay described in the present study was examined. From a practical viewpoint, the preparation of

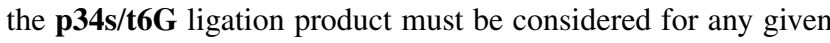
concentration of the substrate compound. Because only limited conditions for the T4 DNA ligase is workable, we prepared a series of DNA solutions of given concentration by diluting a

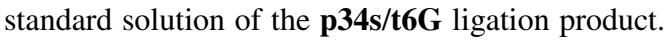

The obtained representative AFM images (Fig. 4) showed certain tendencies in response to concentration. The number of grains on the $\mathrm{Au}$ that are single p34s-t6G molecules clearly decreased as the concentration of the ODN in the test solution was decreased. While the molecules are decentrally organized down to $5 \times 10^{-11} \mathrm{M}$, at lower concentration it becomes impossible to identify individual molecules by simply obtaining the surface images and by watching them. As can be seen in Fig. 4 at those concentrations reappear the sharp edges associated with the $\mathrm{Au}(111)$ terrace structure. In this regard, one may notice that AFM images consist of a set of pixels into which specific height data are encoded. We found that, using this particular feature, simple histogram analysis could allow convenient quantification. ${ }^{21,22}$ Figure 4 explains each frequency distribution fundamentally traces a specific curve typical of a normal distribution. Nonlinear curve fitting assuming a set of multiple Gaussian function peaks was also performed; the results are summarized in Table 2.

These data allow a simple, straightforward interpretation of 

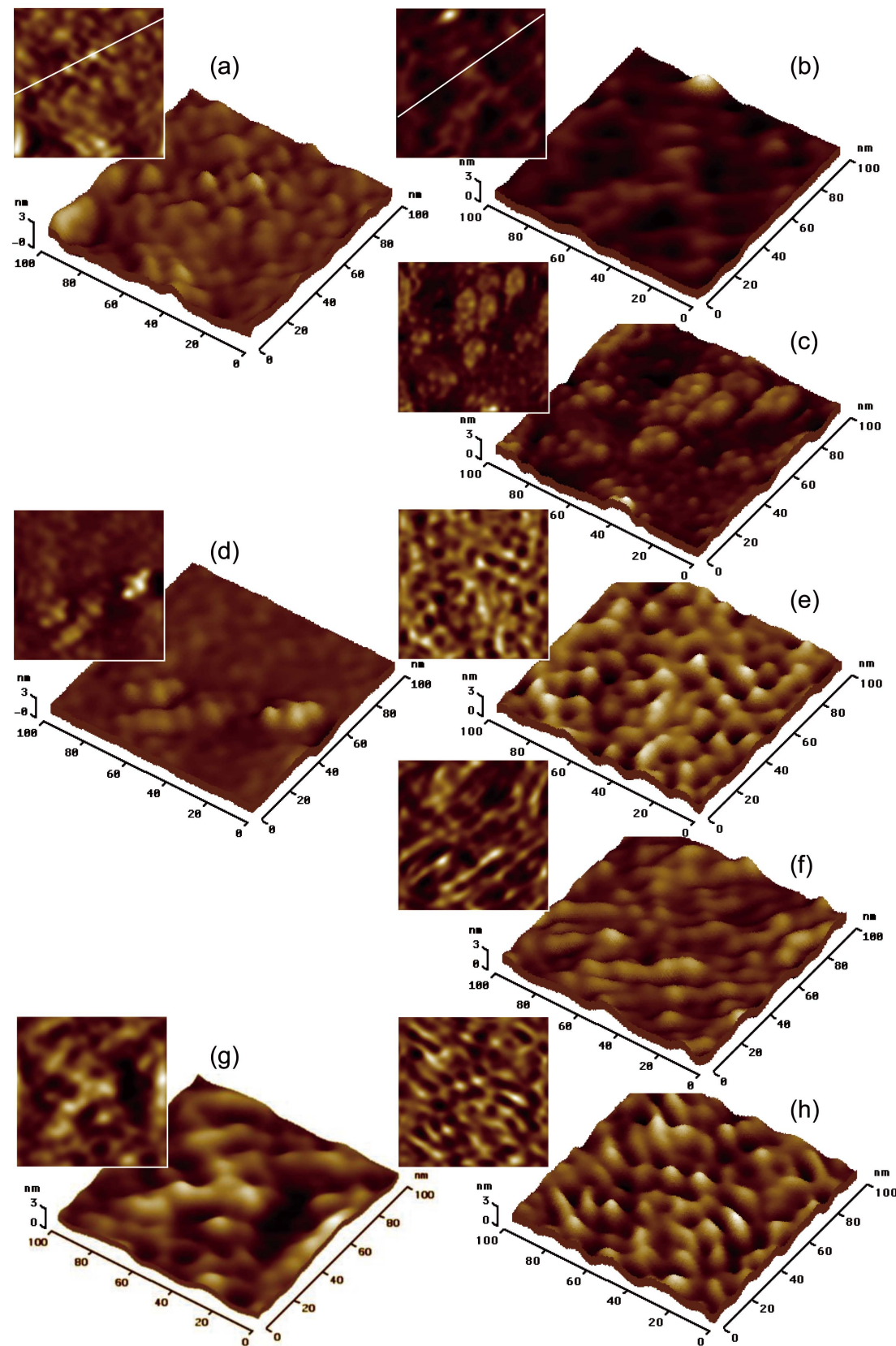

(c)

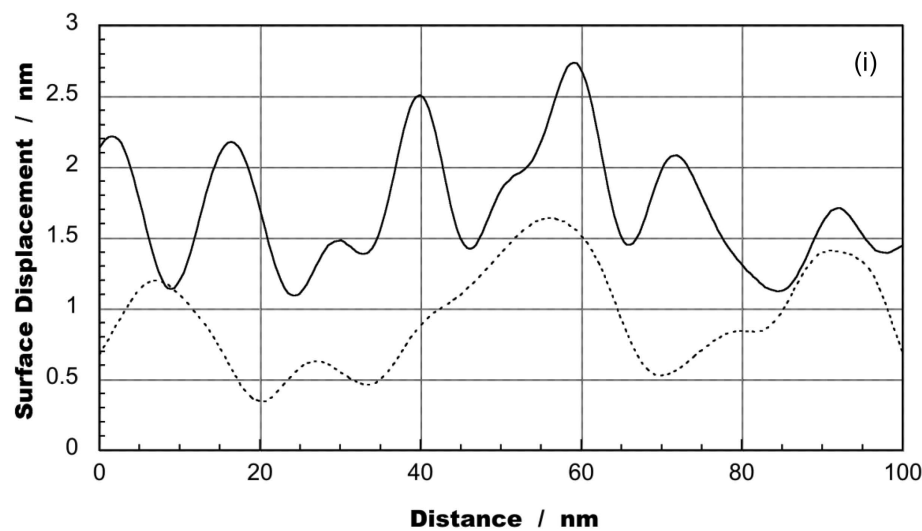

Fig. 3 Topographical images of $\mathrm{Au}(111)$ substrates containing self-assembled $\mathbf{p 3 4 s}$ (a), after hybridization of p34s and $\mathrm{o8}(\mathrm{b})$, control probe of c40s (c), p34s and t6G (d), after ligation of p34s and t6G, and hybridization with $\mathbf{0 8}$ (e), without ligation of p34s and t6G, and hybridization with $\mathbf{0 8}$ (f), p34s and t6A (g), after ligation of p34s and t6A, and hybridization with $\mathbf{0 8}(\mathrm{h})$. In panel (i), the crosssection profiles corresponding to the white lines in (a) and (b) are shown as the solid line and the dotted line, respectively. All images are with numbers that correspond to those in Fig. 1. 

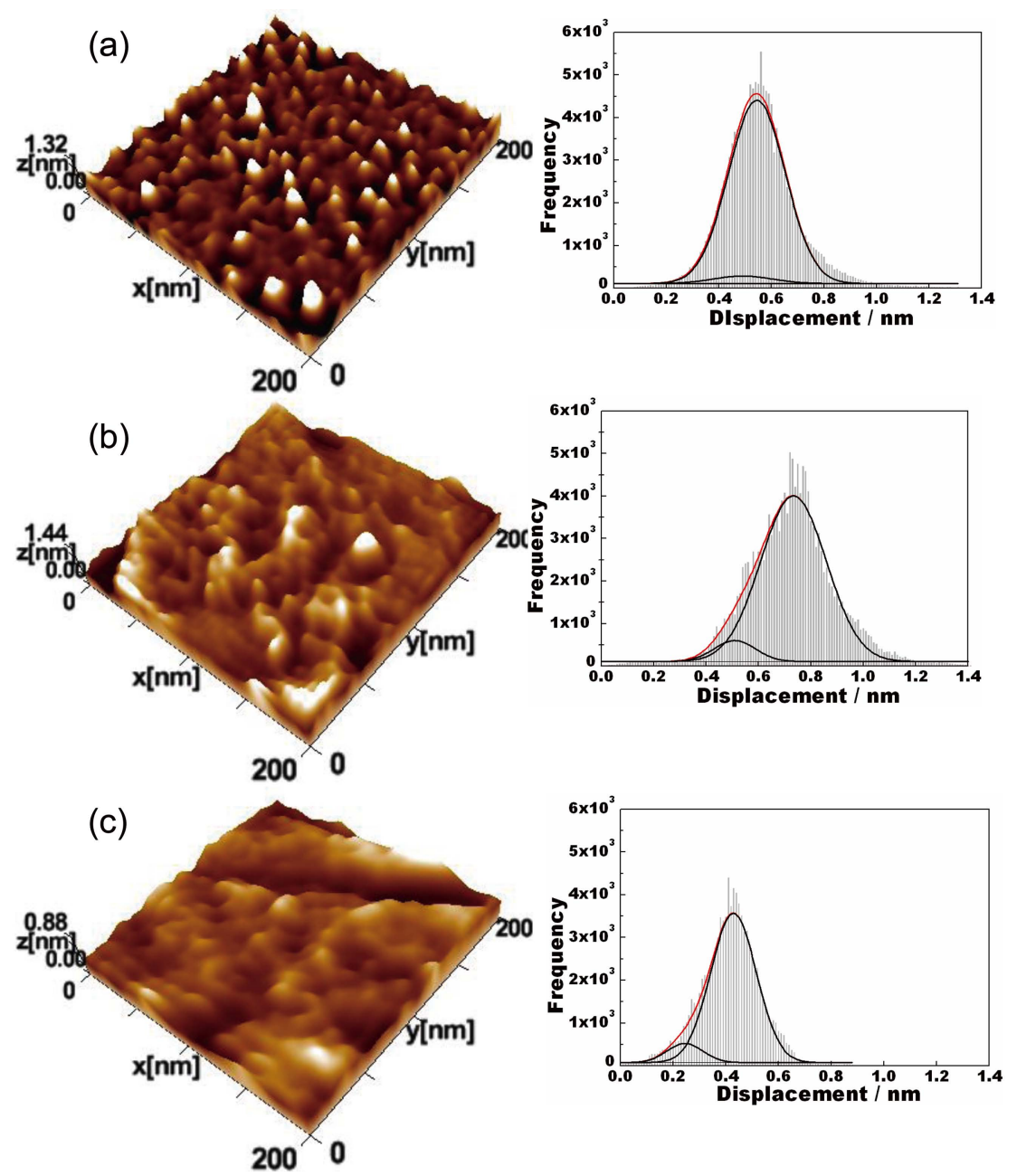

Fig. 4 Changes of the AFM images and the corresponding surface roughness profiles with the decreasing concentration of p34s-t6G obtained at $5 \times 10^{-7} \mathrm{M}(\mathrm{a}), 5 \times 10^{-10} \mathrm{M}(\mathrm{b})$, and $5 \times 10^{-12} \mathrm{M}$ (c).

Table 2 Peak fractions determined by non-linear curve fitting toward the surface roughness profiles

\begin{tabular}{|c|c|c|c|}
\hline \multirow{2}{*}{ Run } & \multirow{2}{*}{ Concentration/M } & \multicolumn{2}{|c|}{$\begin{array}{l}\text { Surface roughness and peak } \\
\text { fraction, } \%\end{array}$} \\
\hline & & $0.2-0.5 \mathrm{~nm}$ & $0.6-0.9 \mathrm{~nm}$ \\
\hline 1 & $5 \times 10^{-7}$ & 3 & 97 \\
\hline 2 & $5 \times 10^{-8}$ & 11 & 89 \\
\hline 3 & $5 \times 10^{-9}$ & - & 100 \\
\hline 4 & $5 \times 10^{-10}$ & 8 & 92 \\
\hline 5 & $5 \times 10^{-11}$ & 100 & - \\
\hline 6 & $5 \times 10^{-12}$ & 100 & - \\
\hline
\end{tabular}

the concentration-dependent surface morphology responses. First, the distribution profile of the surface morphology clearly specifies a two-component model; one is a heavily-textured component and the other is a smaller one that is nearly identical to that of the bare $\mathrm{Au}(111)$ substrate. The former fraction primarily appears at higher concentration, and thus can be attributed to p34s-t6G. As the concentration of p34s-t6G is decreased, the surface coverage of the granular matter reduces, and at $5 \times 10^{-10} \mathrm{M}$ the Au surface becomes exposed in part. At concentrations of less than $5 \times 10^{-11} \mathrm{M}$, most of the Au surface is bare. Therefore, p34s-t6G is no longer able to maintain the vertically-oriented surface structure; it may take the horizontal orientation due to the non-specific interaction between the nucleobases and $\mathrm{Au}$. In this instance, we were not able to discriminate them from the surface roughness for the substrate by simple histogram analysis. Consequently, we conclude that the detection limit should lie at around $5 \times 10^{-10} \mathrm{M}$. Preparation of the AFM specimens only requires a very small volume of test solution, typically $10 \mu \mathrm{L}$ or less. An extremely high detection sensitivity of $5 \mathrm{fmol}$ was achieved for the SNP assay. Although this sensitivity falls short to that of ELISA, it is on par with simple fluorophore-labeling methods.

\section{Conclusions}

DNA, as a genetic material relevant to our health, has become a material central to biosensing. Until now, the molecular labeling methods have developed various DNA biosensing techniques. Simultaneously, recent developments in nanotechnology, particularly AFM, have been anticipated to offer the potential of advanced biosensing. We have achieved for the first time a molecular-observation method for detecting K-ras SNPs by incorporating the hairpin-to-linear conformational change into a simple ligase assay. Currently, SNP typing is made by 
hybridization assay using allele-specific oligonucleotide probes, ${ }^{23-25}$ or by enzymic reactions including DNA ligase (oligonucleotide ligation assay), ${ }^{26,27}$ DNA polymerase (primer extension assay ${ }^{28-30}$ or TaqManPCR method), ${ }^{31,32}$ and also endnuclease (invader assay). ${ }^{33-35}$ In the present method, T4 DNA ligase plays the key role in mismatch recognition, and therefore applications are limited to the terminal SNP samples as in analogy with the most enzymic system. However, a potentially label-free method of SNP detection is enabled by observing the loop-to-linear macromolecular conformational change. Current AFM is rather sophisticated technique and requires great care both of which limit general use. However, recent high-speed $\mathrm{AFMs}^{36}$ may help to improve the present situation enabling a combination with any parallelized device including DNA microarrays. Essential are verifications regarding the usefulness and applicability to the real samples that are lower in concentration and typically a mixture; amplification by polymerase chain reaction may clear the former problem and we are now seriously looking at the later issue. Results will be reported elsewhere.

\section{Acknowledgements}

We thank Prof. A. Takahara and Dr. M. Kobayashi, Kyushu University, for access to the ellipsometer. We also thanks to the Center of Advanced Instrumental Analysis, Kyushu University for use of the FTIR spectrometer. K. N. acknowledges financial support from the Precursory Research for Embryonic Science and Technology, the Japan Science and Technology Agency and from a Grant-in-Aid for Scientific Research from the Ministry of Education, Culture, Sports, Science and Technology (MEXT), Japan. This work was also supported by the Kyushu University Global COE Program-Science for Future Molecular Systems, and Nanotechnology-Support Network Program in Kyushu Area (MEXT).

\section{Supporting Information}

Fluorescence energy-transfer measurements inherently confirmed the working principle. This material is available free of charge on the Web at http://www.jsac.or.jp/analsci/.

\section{References}

1. T. A. Brown, "Genomes 3", 2006, Chaps. 3 and 4, Garland Science, New York.

2. M. Endo and H. Sugiyama, ChemBioChem., 2009, 10, 2420.

3. S. Tyagi and F. R. Kramer, Nat. Biotechnol., 1996, 14, 303.

4. A. S. Piatek, S. Tyagi, A. C. Pol, A. Telenti, L. P. Miller, F. R. Kramer, and D. Alland, Nat. Biotechnol., 1998, 16, 359.

5. H. Du, C. M. Strohsahl, J. Camera, B. L. Miller, and T. D. Krauss, J. Am. Chem. Soc., 2005, 127, 7932.

6. M. P. Raphael, J. A. Christodoulides, S. N. Qadri, S. A. Qadri, M. M. Miller, L. K. Kurihara, and J. M. Byers, Biosens. Bioelectron., 2008, 24, 888.

7. S. A. E. Marras, F. R. Kramer, and S. Tyagi, Genet. Anal. Biomol. Eng., 1999, 14, 151.

8. A. Tsourkas, M. A. Behlke, Y. Xu, and G. Bao, Anal. Chem, 2003, 75, 3697.

9. Y. Wu, C. J. Yang, L. L. Moroz, and W. Tan, Anal. Chem., 2008, 80, 3025.
10. C. B. Swearingen, D. P. Wernette, D. M. Cropek, Y. Lu, J. V. Sweedler, and P. W. Bohn, Anal. Chem., 2005, 77, 442.

11. R. Y. Lai, E. T. Lagally, S. H. Lee, H. T. Soh, K. W. Plaxco, and A. J. Heeger, Proc. Natl. Acad. Sci. U. S. A., 2006, 103, 4017.

12. M. Zuker, Nucleic Acids Res., 2003, 31, 3406.

13. W. A. Kibbe, Nucleic Acids Res., 2007, 35, W43.

14. J. B. Schlenoff, M. Li, and H. Ly, J. Am. Chem. Soc., 1995, 117, 12528

15. K. Nakano, T. Yoshitake, Y. Yamashita, and E. F. Bowden, Langmuir, 2007, 23, 6270.

16. Image $S X M$, S. D. Barrett, 2008, http://www.ImageSXM. org.uk/.

17. K. Nakano, H. Yamanouchi, H. Yoshinaga, N. Soh, and T. Imato, Chem. Commun., 2010, 46, 5683.

18. T. Ihara, M. Nakayama, M. Murata, K. Nakano, and M. Maeda, Chem. Commun., 1997, 1609.

19. M. Nakayama, T. Ihara, K. Nakano, and M. Maeda, Talanta, 2002, 56, 857.

20. W. Saenger, "Principles of Nucleic Acid Structure", 1984 Springer-Verlag, New York, 242.

21. K. Nakano, Y. Katasumi, N. Soh, and T. Imato, Bull. Chem. Soc. Jpn., 2010, 3, 273.

22. K. Nakano, H. Matsunaga, M. Murata, N. Soh, and T. Imato, Anal. Sci., 2009, 25, 993.

23. J. G. Hacia, B. Sun, N. Hunt, K. Edgemon, D. Mosbrook, C. Robbins, S. P. A. Fodor, D. A. Tagle, and F. S. Collins, Genome Res., 1998, 8, 1245.

24. D. G. Wang, J. B. Fan, C. J. Siao, A. Berno, P. Young, R. Sapolsky, G. Ghandour, N. Perkins, E. Winchester, J. Spencer, L. Kruglyak, L. Stein, L. Hsie, T. Topaloglou, E. Hubbell, E. Robinson, M. Mittmann, M. S. Morris, N. Shen, D. Kilburn, J. Rioux, C. Nusbaum, S. Rozen, T. J. Hudson, R. Lipshutz, M. Chee, and E. S. Lander, Science, 1998, 280, 1077.

25. S. Tyagi, D. P. Bratu, and F. R. Kramer, Nat. Biotechnol., 1998, 16, 49.

26. X. Chen, K. J. Livak, and P. Y. Kwok, Genome Res., 1998, 8, 549.

27. M. A. Iannone, J. D. Taylor, J. Chen, M. S. Li, P. Rivers, K. A. Slentz-Kesler, and M. P. Weiner, Cytometry, 2000, 39, 131.

28. X. Chen, B. Zehnbauer, A. Gnirke, and P. Y. Kwok, Proc. Natl. Acad. Sci. U. S. A., 1997, 94, 10756.

29. J. B. Fan, X. Chen, M. K. Halushka, A. Berno, X. Huang, T. Ryder, R. J. Lipshutz, D. J. Lockhart, and A. Chakravarti, Genome Res., 2000, 10, 853.

30. J. Chen, M. A. Iannone, M. S. Li, J. D. Taylor, P. Rivers, A. J. Nelsen, K. A. Slentz-Kesler, A. Roses, and M. P. Weiner, Genome Res., 2000, 10, 549.

31. T. Morris, B. Robertson, and M. Gallagher, J. Clin. Microbiol., 1996, 34, 2933.

32. K. J. Livak, J. Marmaro, and J. A. Todd, Nat. Genet., 1995, 9, 341.

33. V. Lyamichev, A. L. Mast, J. G. Hall, J. R. Prudent, M. W. Kaiser, T. Takova, R. W. Kwiatkowski, T. J. Sander, M. de Aruda, D. A. Arco, B. P. Neri, and M. A. D. Brow, Nat. Biotechnol., 1999, 17, 292.

34. V. Lyamichev, M. A. D. Brow, V. E. Varvel, and E. Dahlberg, Proc. Natl. Acad. Sci. U. S. A., 1999, 96, 6143.

35. T. J. Griffin, J. G. Hall, J. R. Prudent, and L. M. Smith, Proc. Natl. Acad. Sci. U. S. A., 1999, 96, 6301.

36. T. Ando, T. Uchihashi, and T. Fukuma, Prog. Surf. Sci., 2008, 83, 337. 